\title{
Representações da tríplice escolar sobre parasitoses intestinais na zona rural de Paulo Afonso - Bahia
}

\author{
Maria Tairla Viana Gonçalves \\ Graduada em Licenciatura Plena em Ciências Biológicas, Universidade do Estado da Bahia - UNEB, \\ Departamento de Educação, Campus VIII, Paulo Afonso - BA \\ $\square$ tairlagoncalves22@gmail.com
}

Deyvison Rhuan Vasco-dos-Santos

Doutorando em Biologia Parasitária, Instituto Oswaldo Cruz - IOC/ Fiocruz, no Laboratório de Inovações em Terapias, Ensino e Bioprodutos, Rio de Janeiro - RJ

$\square$ deyvisonvs@gmail.com

\section{Erika dos Santos Nunes}

Doutora em Ciências Biológicas, Universidade Federal de Pernambuco

Docente da Universidade do Estado da Bahia - UNEB,

Departamento de Educação, Campus VIII, Paulo Afonso - BA

$\square$ erika.santosnunes@hotmail.com

Recebido em 1 de junho de 2020

Aceito em 2 de julho de 2020

\begin{abstract}
Resumo:
O estudo das representações acerca das parasitoses intestinais auxilia na compreensão dos casos de adoecimentos e contribuem para tomada de ações voltadas a melhoria da saúde coletiva, especialmente da população pediátrica. Nesse contexto, a pesquisa teve como objetivo investigar as representações da tríplice escolar (discentes, responsáveis e docentes) de seis escolas municipais da zona rural de Paulo Afonso - Bahia sobre enteroparasitoses. Para coleta de dados, foram administrados formulários semiestruturados específicos para cada seguimento da pesquisa e as informações categorizadas e analisadas com base na literatura especializada. No total, 63 discentes, 55 responsáveis e 9 docentes foram entrevistados. Referente aos agentes etiológicos, os discentes $(68,2 \%)$ e pais $(60,0 \%)$ não detinham conhecimento quanto a definição ou nomeação adequada, enquanto, os docentes $(77,4 \%)$, sim. Quanto a transmissão, discentes $(55,5 \%)$ e pais $(60,0 \%)$ não souberam responder ou responderam incorretamente, dentre os docentes $(66,7 \%)$ responderam corretamente e $(22,2 \%)$ parcialmente correto. Acerca do hábitat, $(80,9 \%)$ dos alunos, $(69,1 \%)$ dos pais e $(88,9 \%)$ dos professores responderam corretamente, sendo os órgãos mais citados: intestino grosso e delgado. Sobre os sintomas, discentes $(22,2 \%)$ e pais $(36,4 \%)$ reportaram dor de barriga e os docentes $(66,7 \%)$ falta de apetite. Quanto a prevenção, $(25,4 \%)$ dos discentes não souberam responder e $(19,0 \%)$ responderam incorretamente, enquanto $(50,9 \%)$ dos pais e $(88,9 \%)$ dos docentes responderam corretamente. Considerando as fragilidades sobre o conceito e ciclo de vida dos parasitos, torna-se necessário a realização de intervenções educativas, visando formar multiplicadores de conhecimento e minimizar os casos de infecções em prol da qualidade de vida individual e coletiva.

Palavras-chave: Percepções sociais, Ecologia Médica, Etnoparasitologia, Educação em Saúde, Parasitologia humana.
\end{abstract}




\title{
Representations of the school triple about intestinal parasites in the rural area of Paulo Afonso - Bahia
}

\begin{abstract}
:
The study about intestinal parasites representations help to understand the cases of sickness and contribute to taking actions aimed at improving collective health, especially for the pediatric population. In this context, the research aimed to investigate the knowledge of the triple school (students, guardians and teachers) of six municipal schools in the rural area of Paulo Afonso - Bahia about intestinal parasites. For data collection, specific semi-structured forms were administered for each segment of the research and the information categorized and analyzed based on the specialized literature. In total, 63 students, 55 guardians and 9 teachers were interviewed. Regarding etiologic agents, students (68.2\%) and parents (60.0\%) do not detect knowledge about the proper definition or appointment, while teachers (77.4\%), yes. As for transmission, students (55.5\%) and parents (60.0\%) do not know how to answer or answer incorrectly, among the documents (66.7\%) answer correctly and (22.2\%) are correctly selected. Regarding habitat, (80.9\%) of students, $(69.1 \%)$ of parents and (88.9\%) of teachers responded correctly, with the most cited organs: large and small intestine. Regarding the symptoms, students (22.2\%) and parents (36.4\%) reported abdominal pain and teachers (66.7\%) lack of appetite. Regarding prevention, (25.4\%) of students did not know how to answer and (19.0\%) answered incorrectly, while (50.9\%) of parents and (88.9\%) of teachers responded correctly. Considering the weaknesses on the concept and life cycle of the parasites, it is necessary the realization of educational interventions, aiming at forming knowledge multipliers and minimizing cases of infections in favor of individual and collective quality of life.
\end{abstract}

Keywords: Social perceptions, Medical Ecology, Ethnoparasitology, Health education, Human parasitology.

\section{Representaciones del triple escolar sobre parasitos intestinales en el área rural de Paulo Afonso - Bahia}

\section{Resumen:}

El estudio de las representaciones sobre parásitos intestinales ayuda a comprender los casos de enfermedad y contribuye a tomar medidas destinadas a mejorar la salud colectiva, especialmente para la población pediátrica. En este contexto, la investigación tuvo como objetivo investigar las representaciones de la triple escuela (estudiantes, padres / tutores y maestros) de seis escuelas municipales en la zona rural de Paulo Afonso - Bahía sobre enteroparasitosis. Para la recolección de datos, se administraron formas semiestructuradas específicas para cada segmento de la investigación y la información clasificada y analizada en base a la literatura especializada. En total, se entrevistó a 63 estudiantes, 55 padres y 9 maestros. Con respecto a los agentes etiológicos, los estudiantes (68.2\%) y los padres (60.0\%) no tenían conocimiento sobre la definición o nominación adecuada, mientras que los maestros (77.4\%) sí. En cuanto a la transmisión, los estudiantes (55.5\%) y los padres (60.0\%) no sabían cómo responder o respondieron incorrectamente, entre los maestros (66.7\%) respondieron correctamente y $(22.2 \%)$ parcialmente correcto. Con respecto al hábitat, $(80.9 \%)$ de los estudiantes, (69.1\%) de los padres y (88.9\%) de los maestros respondieron correctamente, con los órganos más citados: intestino grueso y delgado. Con respecto a los síntomas, los estudiantes (22.2\%) y los padres (36.4\%) informaron dolor abdominal y la falta de apetito de los maestros (66.7\%). Con respecto a la prevención, (25.4\%) de los estudiantes no sabían cómo responder y (19.0\%) respondieron incorrectamente, mientras que (50.9\%) de los padres y (88.9\%) de los maestros respondieron correctamente. Considerando las debilidades sobre el concepto y el ciclo de vida de los parásitos, es necesario llevar a cabo intervenciones educativas, con el objetivo de formar multiplicadores de conocimiento y minimizar los casos de infecciones a favor de la calidad de vida individual y colectiva. Palabras clave: Percepciones sociales, Ecologia medica, Etnoparasitología, Educación en salud, Parasitología humana. 


\section{INTRODUÇÃO}

As representações sociais constituem uma modalidade de conhecimento necessária para entender os comportamentos dos indivíduos, pois permitem a construção de mapas de informações, configurados a partir de ideias ou conhecimentos preexistentes, com influência de aspectos fisiológicos, psicológicos e culturais (MOSCOVICI, 1978; ALBUQUERQUE, 2014). Concernente aos processos de saúde e adoecimento, os estudos das representações sociais auxiliam na compreensão das matrizes culturais que expressam significados e ações relativas ao itinerário terapêutico, possibilitando identificar os fatores determinantes para casos de adoecimento, incorporação de hábitos saudáveis e entendimento de como as ações humanas influenciam a ocorrência de infecções, a exemplo das parasitárias (GAZZINELLI et al., 2005; NUNES; CUNHA; JÚNIOR, 2006; ALVES, 2015).

No âmbito escolar, pesquisas acerca dos saberes de alunos, pais e funcionários sobre parasitoses intestinais, evidenciam que a ausência ou fragilidades no conhecimento são fatores de risco para aquisição de parasitos intestinais, uma vez que refletem em seus comportamentos (MONROE et al., 2013; SIQUEIRA et al., 2016). No estudo de Oliveira et al. (2019) com estudantes de áreas periurbanas de um município do Maranhão, foram realizadas investigações acerca dos costumes e conhecimentos, bem como, fizeram análise parasitológica, constatando que 90,9\% das crianças parasitadas tinham o costume de andar descalças.

As enteroparasitoses constituem um grave problema de saúde pública mundial, acometendo principalmente a população infantil, devido aos princípios básicos de higiene fragilizados, além de possuírem um sistema imunológico em desenvolvimento (ALMEIDA; CARVALHO; MORENO, 2017; BRAGAGNOLLO et al., 2018; FARIA et al., 2019). Inquéritos realizados entre escolares de comunidades rurais constataram elevadas prevalências, sendo o Ascaris lumbricoides Linnaeus, 1758, Trichuris trichiura Linnaeus, 1771 e Ancilostoma duodenale Dubini, 1843, os agentes infecciosos mais encontrados (FONSECA et al., 2010; SILVA; CARVALHO; FIRMO, 2016). Este quadro pode estar associado às dificuldades de acesso aos serviços de saúde e fragilidades de informações acerca das medidas preventivas, tornando-se necessário a realização de intervenções educativas (MONTEIRO et al., 2009; SILVA; CARVALHO; FIRMO, 2016).

A ocorrência de parasitoses intestinais na população infantil, são por vezes, 
decorrentes da falta de conhecimento da família que participa desde cedo da tomada de decisões das crianças (MONTEIRO et al., 2009). Portanto, torna-se relevante a realização de atividades preventivas no contexto familiar, para instruir as crianças e estimular a adoção de comportamentos que favoreçam a qualidade de vida (BARBOSA et al., 2013). Segundo Costa e Souza (2019), os professores também possuem papel essencial na mediação de atividades de saúde, por estarem em constante contato com os alunos e conhecer a realidade socio-cultural deste público (BRAGAGNOLLO et al., 2017).

Quando o enfoque é a educação para a saúde, a escola torna-se um importante centro de multiplicadores de conhecimento, pois é o local favorável para problematização, mediação e investigação de conceitos, incorporação de bons hábitos, valores e atitudes (MONROE et al., 2013; LOPES; MELLO, 2014). Desse modo, o desenvolvimento de pesquisas acerca do conhecimento dos sujeitos envolvidos no processo ensino-aprendizagem em saúde, são essenciais no fornecimento de subsídios para construção de melhores programas educativos (SIQUEIRA et al., 2016).

Diante da importância das pesquisas sobre representações para compreender a relação entre as concepções sociais e infecções parasitárias, o presente trabalho teve como objetivo investigar o conhecimento da tríplice escolar (discentes, responsáveis e docentes) de escolas municipais da zona rural de Paulo Afonso - BA, acerca das parasitoses intestinais.

\section{METODOLOGIA}

\section{Área de estudo}

O estudo foi realizado na área rural do Município de Paulo Afonso - Bahia, localizado à $460 \mathrm{~km}$ da capital do estado, Salvador, especificamente sob as coordenadas 9²4'27.34"S e 38²'52.00"W. A cidade apresenta uma população estimada de 108.396 habitantes, com aproximadamente 93.404 na zona urbana e 14.992 na zona rural (IBGE, 2010; PREFEITURA MUNICIPAL DE PAULO AFONSO, 2014). 
Para realização do trabalho foram selecionadas seis escolas municipais (Figura 1): João Fernandes de Souza, Pedro Januário dos Santos, Manoel Pereira, Gilberto de Barros Pedrosa, Rui Barbosa e Antônio Ramalho, localizadas nas comunidades Açude, Caiçara II, Salgadinho, Vila Matias, Caiçara I e Arrasta Pé, respectivamente, considerando a viabilidade de acesso.

Figura 1. Localização das seis escolas municipais da zona rural de Paulo Afonso, Bahia, Brasil, participantes do estudo.

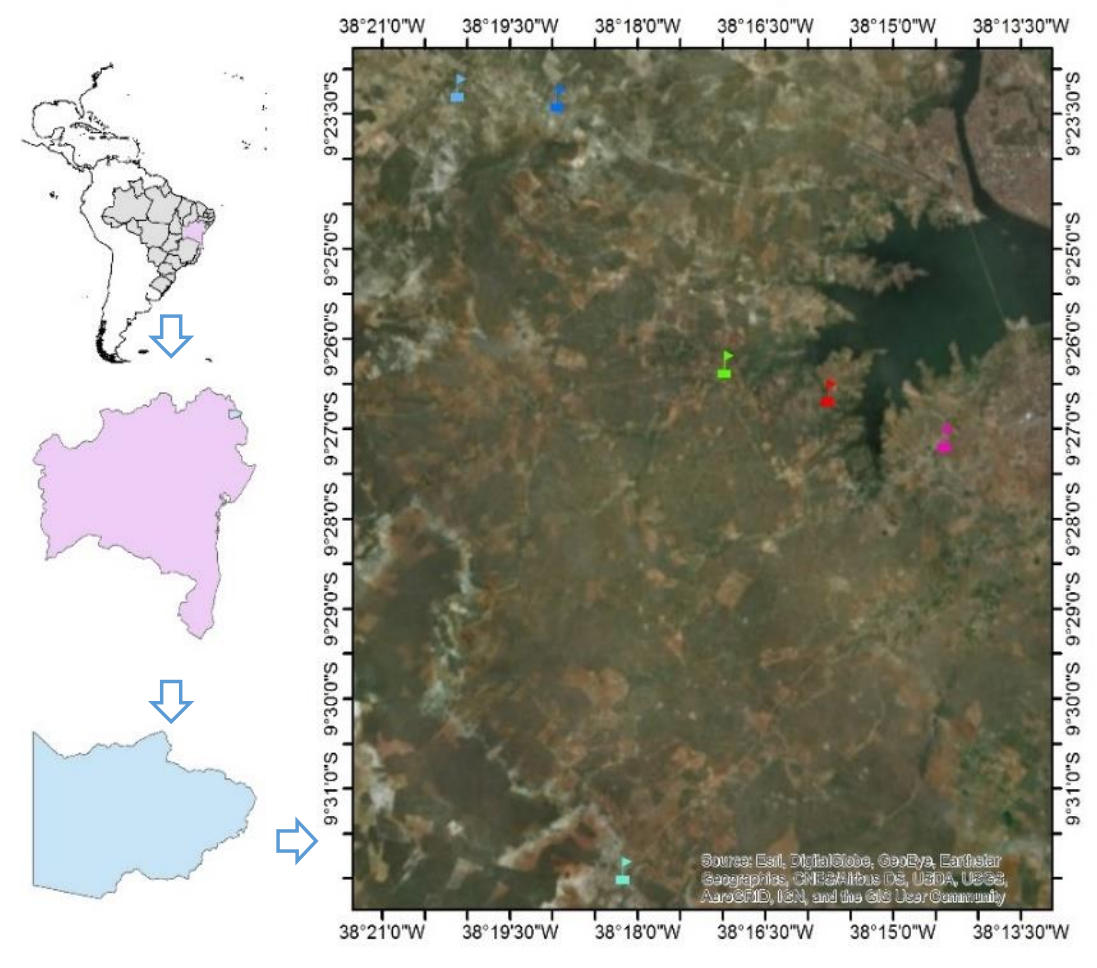

Fonte: Própria.

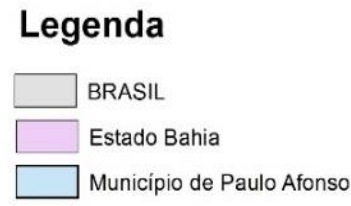

\section{Povoados}

- Arrasta pé Açude Caiçara I Caiçara II Salgadinho Vila Matias

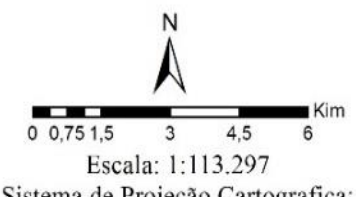

Sistema de Projeção Cartografica: SIRGAS - 2000 Base Cartografica: IBGE, 2006 .

\section{Aspectos éticos da pesquisa e seleção dos participantes}

A pesquisa foi aprovada pelo Comitê de Ética em Pesquisa com Seres Humanos (CEP) da Universidade do Estado da Bahia (UNEB) sob parecer de $n^{\circ} 1.969 .897$, e autorizado pela Secretaria de Educação da Prefeitura Municipal de Paulo Afonso - BA por meio da Carta de Anuência. Inicialmente foram realizadas reuniões nas escolas com os responsáveis e professores para apresentar os objetivos, riscos e benefícios do estudo e convidá-los a participar. 
À participação dos informantes foi condicionada pela assinatura do Termo de Consentimento Livre Esclarecido (TCLE) do adulto e dos menores sob suas responsabilidades. Ademais, às crianças autorizadas por seus responsáveis, assinaram o Termo de Assentimento do Menor (TAM). O trabalho foi realizado com discentes do $1^{o}$ ao $5^{\circ}$ ano do Ensino Fundamental I, com faixa etária de seis a dez anos de idade (MINISTÉRIO DA EDUCAÇÃO, 2013), por constituírem um grupo suscetível à aquisição de parasitos intestinais (BRAGAGNOLLO et al., 2018).

\section{Coleta e análise de dados}

Foram realizadas entrevistas semiestruturados (BERNARD, 2006), utilizando formulários adaptados de Mello et al. (1988), específicos para cada um dos seguimentos incluídos no trabalho (discentes, responsáveis e docentes), acerca do perfil técnicoparasitológico (conceito, transmissão, habitat, sintomas e prevenção). As informações foram tabuladas no Microsoft Excel ${ }^{\circledR}$ 2010, analisadas de acordo com Neves et al. (2018) e categorizadas como corretas (respostas sem erros), parcialmente corretas (respostas com informações certas e erradas), incorretas (respostas apenas com erros) e não soube responder.

\section{RESULTADOS e DISCUSSÃo}

Um total de 127 pessoas foram entrevistadas, sendo 49,6\% discentes, 43,3\% responsáveis e 7,1\% docentes. A avaliação e frequência das múltiplas respostas acerca das representações parasitológicas são apresentadas nas tabelas 1 e 2 . 


\section{Representações dos discentes}

Quando questionados sobre o conceito de vermes, 49,2\% não souberam responder, $19,0 \%$ responderam incorretamente e $31,7 \%$ corretamente. Os nomes mais citados foram bicho (14,3\%), doença, lagarta (4,7\% cada) e minhoca (3,2\%). o conhecimento dos vocábulos pode estar associado aos casos de adoecimento, conforme verificado por Moura et al. (2018) com estudantes de Pelotas - RS, cujo a maioria (54,2\%) afirmou saber o que são parasitos por terem expelido nas fezes quando infectados. Com relação, aos termos minhoca e lagarta, há a possibilidade de estarem relacionados as características morfológicas dos helmintos: corpo mole, esbranquiçado e alongado (NEVES, 2009). Dados semelhantes foram observados na investigação realizada por Malafaia et al. (2013) com alunos de uma escola pública de Urutaí $\mathrm{GO}$, os quais desconheciam $(83,8 \%)$ ou conheciam muito pouco $(7,5 \%)$ o nome dos agentes etiológicos que causam verminoses.

Com relação a transmissão, 35,6\% dos discentes responderam corretamente, mencionando principalmente o contato com o solo. Rivero et al. (2017) a partir da análise de desenhos feitos por escolares sobre verminoses, constataram que o solo também foi a fonte de infecção mais representada (37,0\%). Em contrapartida, 31,7\% dos discentes não souberam responder e 23,8\% responderam incorretamente, similar aos resultados de Ahmed et al. (2017) com estudantes de Asmara - Eritreia (África), que a maioria $(84,1 \%)$ também desconhecia as vias de contaminação.

Sobre o habitat e os sintomas, $80,9 \%$ e $61,9 \%$ respectivamente, responderam corretamente. Os discentes, possivelmente, relacionaram a localização do parasito com o sintoma, por exemplo: I) intestino grosso e delgado (84,1\% cada) com dor de barriga $(22,2 \%)$; vômito (7,9\%), falta de apetite (1,6\%); e II) cérebro (9,5\%) com dor de cabeça (7,9\%). A correlação localização-sintoma é corroborado no trabalho de Siqueira et al. (2016) com estudantes de Niterói - RJ, que citaram intestino $(47,5 \%)$ e dor de barriga $(45,1 \%)$ como principal localização e sintoma, respectivamente. 
Quanto a prevenção, 3,2\% responderam parcialmente correto, 19,0\% incorretamente, $25,4 \%$ não souberam responder e a maior parte $(52,4 \%)$ respondeu corretamente mencionando evitar contato com solo (19,0\%), lavar as mãos (14,3\%), ter higiene (9,5\%) e outras maneiras $(38,1 \%)$. Estas informações assemelham-se com os resultados obtidos por Barbosa et al. (2013) entre alunos da zona rural de Serra Talhada - PE, onde as medidas profiláticas mais citadas foram não andar descalços, lavar as mãos e ter higiene com alimentos e água.

Em uma pesquisa conduzida por Vivas et al. (2010) entre alunos da área rural em Angolela - Etiópia (África) acerca dos hábitos de higiene, 99,0\% relataram lavar as mãos e $36,2 \%$ afirmaram utilizar sabão, ao contrário dos dados encontrados nesse estudo, em que os discentes não informaram como higienizam as mãos. De acordo com Ahmed et al. (2017), a correta lavagem das mãos antes das refeições e após utilizar o banheiro intervém na transmissão oro-fecal das parasitoses e a sua ausência, consequentemente, pode aumentar o risco de infecções parasitárias. 
Tabela 1 - Saberes da tríplice escolar (discentes, responsáveis e docentes) de seis escolas municipais da zona rural de Paulo Afonso - Bahia acerca das $\mathrm{F}$ intestinais. E.1: João Fernandes de Souza; E.2: Pedro Januário dos Santos; E.3: Manoel Pereira; E.4: Antônio Ramalho; E.5: Rui Barbosa e E.6: Gilberto Pedrosa. I: Respostas corretas; II: Respostas parcialmente corretas; III: Respostas incorretas; IV: Não souberam responder.

\begin{tabular}{|c|c|c|c|c|c|c|c|c|c|c|c|c|c|c|c|c|c|c|c|c|c|c|}
\hline \multirow{2}{*}{ Questões } & \multirow{2}{*}{$\begin{array}{c}\text { Respostas } \\
\text { (\%) }\end{array}$} & \multicolumn{7}{|c|}{ ntes } & \multicolumn{7}{|c|}{ Responsáveis } & \multicolumn{7}{|c|}{ Docentes } \\
\hline & & E.1 & E.2 & E.3 & E.4 & E.5 & E.6 & Total & E.1 & E.2 & E.3 & E.4 & E.5 & E.6 & Total & E.1 & E.2 & E.3 & E.4 & E.5 & E.6 & Total \\
\hline \multirow{4}{*}{ Conceito } & $\mathrm{I}$ & 6,7 & 50,0 & - & - & - & - & 9,5 & 14,3 & - & 60,0 & - & 11,1 & 23,1 & 16,4 & 100,0 & - & 100,0 & 50,0 & 100,0 & - & 55,6 \\
\hline & II & 26,7 & - & 40,0 & - & 45,4 & 18,8 & 22,2 & 21,4 & 37,5 & 20,0 & 50,0 & 22,2 & 7,6 & 23,6 & - & - & - & 50,0 & - & 50,0 & 22,2 \\
\hline & III & 20,0 & 30,0 & - & 16,7 & 9,1 & 25,0 & 19,0 & 28,6 & 12,5 & 20,0 & 16,7 & 22,2 & 53,8 & 29,1 & - & 100,0 & - & - & - & 50,0 & 22,2 \\
\hline & IV & 46,6 & 20,0 & 60,0 & 83,3 & 45,4 & 56,2 & 49,2 & 35,7 & 50,0 & - & 33,3 & 44,4 & 15,4 & 30,9 & - & - & - & - & - & - & - \\
\hline \multirow{4}{*}{ Transmissão } & I & 33,3 & 70,0 & 60,0 & - & 9,1 & 43,7 & 36,5 & 14,3 & - & 80,0 & 66,7 & 11,1 & 30,8 & 27,3 & 100,0 & 100,0 & 100,0 & - & 100,0 & 50,0 & 66,7 \\
\hline & II & 6,7 & 10,0 & - & - & 9,1 & 12,5 & 7,9 & 7,1 & - & - & - & 22,2 & 7,7 & 7,3 & - & - & - & 50,0 & - & 50,0 & 22,2 \\
\hline & III & 20,0 & 20,0 & 20,0 & 16,7 & 45,4 & 18,8 & 23,8 & 7,1 & - & - & - & - & 15,4 & 5,4 & - & - & - & - & - & - & - \\
\hline & IV & 40,0 & - & 20,0 & 83,3 & 36,4 & 25,0 & 31,7 & 71,4 & 100,0 & 20,0 & 33,3 & 66,7 & 46,1 & 60,0 & - & - & - & 50,0 & - & - & 11,1 \\
\hline \multirow{4}{*}{ Habitat } & I & 66,7 & 90,0 & 80,0 & 83,3 & 72,7 & 93,8 & 80,9 & 85,7 & 37,5 & 80,0 & 66,7 & 44,4 & 84,6 & 69,1 & 100,0 & 100,0 & - & 50,0 & 50,0 & 50,0 & 55,6 \\
\hline & II & 20,0 & 10,0 & 20,0 & - & 18,2 & - & 11,1 & 7,1 & - & 20,0 & 16,6 & 22,2 & - & 9,1 & - & - & - & 50,0 & 50,0 & 50,0 & 33,3 \\
\hline & III & 6,7 & - & - & 16,7 & - & 6,2 & 4,8 & 7,1 & - & - & - & 11,1 & - & 3,6 & - & - & - & - & - & - & - \\
\hline & IV & 6,7 & - & - & - & 9,1 & - & 3,2 & - & 62,5 & - & 16,6 & 22,2 & 15,4 & 18,2 & - & - & 100,0 & - & - & - & 11,1 \\
\hline \multirow{4}{*}{ Sintomas } & I & 46,7 & 50,0 & 100,0 & 16,7 & 72,7 & 81,2 & 61,9 & 71,4 & 37,5 & 100,0 & 83,3 & 77,8 & 61,5 & 69,1 & 100,0 & 100,0 & 100,0 & 100,0 & 100,0 & 100,0 & 100,0 \\
\hline & II & - & 10,0 & - & 33,3 & - & - & 4,8 & 21,4 & 12,5 & - & - & - & 7,7 & 9,1 & - & - & - & - & - & - & - \\
\hline & III & - & - & - & - & - & - & - & 7,1 & - & - & - & - & 7,7 & 3,6 & - & - & - & - & - & - & - \\
\hline & IV & 53,3 & 40,0 & - & 50,0 & 27,3 & 18,8 & 33,3 & - & 50,0 & - & 16,7 & 22,2 & 23,0 & 18,2 & - & - & - & - & - & - & - \\
\hline \multirow{4}{*}{ Prevenção } & I & 73,3 & 80,0 & 80,0 & - & 36,4 & 37,5 & 52,4 & 71,4 & 12,5 & 60,0 & 50,0 & 33,3 & 61,5 & 50,9 & 100,0 & 100,0 & 100,0 & 100,0 & 100,0 & 50,0 & 88,9 \\
\hline & II & - & 10,0 & - & - & 9,1 & - & 3,2 & - & 25,0 & 20,0 & - & 11,1 & 7,6 & 9,1 & - & - & - & - & - & 50,0 & 11,1 \\
\hline & III & 6,7 & - & - & 16,7 & 45,4 & 31,2 & 19,0 & - & - & 20,0 & - & 11,1 & - & 3,6 & - & - & - & - & - & - & - \\
\hline & IV & 20,0 & 10,0 & 20,0 & 83,3 & 9,1 & 31,2 & 25,4 & 28,5 & 62,5 & - & 50,0 & 44,4 & 30,7 & 36,4 & - & - & - & - & - & - & - \\
\hline \multicolumn{2}{|c|}{$\mathrm{N}^{o}$ Total de participantes } & 15 & 10 & 5 & 6 & 11 & 16 & 63 & 14 & 8 & 5 & 6 & 9 & 13 & 55 & 1 & 1 & 1 & 2 & 2 & 2 & 9 \\
\hline
\end{tabular}

Fonte: Elaborado pelos autores 
Tabela 2 - Frequência de múltiplas respostas dos saberes da tríplice escolar (discentes, responsáveis e docentes) de seis escolas municipais da zona rural de Paulo Afonso - Bahia acerca das parasitoses intestinais. E.1: João Fernandes de Souza; E.2: Pedro Januário dos Santos; E.3: Manoel Pereira; E.4: Antônio Ramalho; E.5: Rui Barbosa e E.6: Gilberto de Barros Pedrosa.

\begin{tabular}{|c|c|c|c|c|c|c|c|c|c|c|c|c|c|c|c|c|c|c|c|c|c|c|}
\hline \multirow[b]{2}{*}{ Questões } & \multirow[b]{2}{*}{ Respostas (\%) } & \multicolumn{7}{|c|}{ Discentes } & \multicolumn{8}{|c|}{ Responsáveis } & \multicolumn{6}{|c|}{ Docentes } \\
\hline & & E.1 & E.2 & E.3 & E.4 & E.5 & E.6 & $\begin{array}{c}\text { Tota } \\
1\end{array}$ & E.1 & E.2 & E. 3 & E.4 & E. 5 & E.6 & $\begin{array}{c}\text { Tota } \\
1 \\
\end{array}$ & E.1 & E. 2 & E. 3 & E.4 & E. 5 & E.6 & Total \\
\hline \multirow{4}{*}{ Transmissão } & $\begin{array}{l}\text { Contato com } \\
\text { solo }\end{array}$ & 20,0 & 70,0 & 60,0 & - & 18,2 & 50,0 & 36,5 & - & - & 60,0 & - & - & 15,4 & 9,1 & 100,0 & 100,0 & - & - & - & 50,0 & 33,3 \\
\hline & Doces & 13,3 & - & 20,0 & 16,7 & 36,4 & 18,7 & 17,5 & 7,1 & - & - & - & - & 15,4 & 5,4 & - & - & - & 50,0 & - & - & 11,1 \\
\hline & $\begin{array}{l}\text { Alimentos } \\
\text { contaminados }\end{array}$ & - & - & 20,0 & - & - & - & 1,6 & 21,4 & - & 100,0 & 33,3 & 11,1 & - & 20,0 & 100,0 & 100,0 & 100,0 & - & 100,0 & - & 55,5 \\
\hline & Outros & 26,7 & 60,0 & 80,0 & - & 9,1 & 6,2 & 25,4 & 14,3 & - & - & 83,3 & 55,5 & 23,1 & 27,3 & - & 100,0 & - & 50,0 & 100,0 & 100,0 & 66,7 \\
\hline \multirow{4}{*}{ Habitat } & Cérebro & 6,7 & - & - & - & 18,2 & 25,0 & 9,5 & 7,1 & - & - & 16,7 & - & 7,7 & 5,4 & 100,0 & - & - & - & - & 100,0 & 33,3 \\
\hline & $\begin{array}{c}\text { Intestino } \\
\text { grosso/delgado }\end{array}$ & 86,7 & 80,0 & 80,0 & 100,0 & 81,8 & 81,2 & 84,1 & 85,7 & 37,5 & 80,0 & 50,0 & 44,4 & 76,9 & 65,4 & 100,0 & 100,0 & - & 100,0 & 100,0 & 50,0 & 77,8 \\
\hline & Ânus & 13,3 & 10,0 & - & 16,7 & 9,1 & 6,2 & 9,5 & 7,1 & - & - & - & 11,1 & 7,7 & 5,4 & - & - & - & 50,0 & - & - & 11,1 \\
\hline & Outros & 46,7 & 40,0 & 60,0 & 33,3 & 36,4 & 6,2 & 33,3 & 28,6 & - & 80,0 & 50,0 & 33,3 & 23,1 & 30,9 & - & 100,0 & - & 100,0 & 100,0 & 100,0 & 77,8 \\
\hline \multirow{6}{*}{ Sintomas } & Dor na barriga & 13,3 & 50,0 & 40,0 & - & 27,3 & 12,5 & 22,2 & 28,6 & 37,5 & 60,0 & 33,3 & 44,4 & 30,8 & 36,4 & 100,0 & 100,0 & 100,0 & - & - & - & 33,3 \\
\hline & Dor na cabeça & 6,7 & 20,0 & - & - & - & 12,5 & 7,9 & - & - & 20,0 & - & - & 7,7 & 3,6 & - & - & - & - & - & - & - \\
\hline & $\begin{array}{l}\text { Vontade de } \\
\text { comer doces }\end{array}$ & - & - & - & 16,7 & - & - & 1,6 & 14,3 & 12,5 & 12,5 & - & - & 7,7 & 9,1 & - & - & 100,0 & - & - & - & 11,1 \\
\hline & Falta de apetite & - & - & 20,0 & - & - & - & 1,6 & 42,8 & 12,5 & 40,0 & 16,7 & 44,4 & - & 25,4 & 100,0 & 100,0 & 100,0 & 50,0 & 50,0 & 50,0 & 66,7 \\
\hline & Manchas & 6,7 & - & - & 16,7 & - & - & 3,2 & 14,3 & 37,5 & 20,0 & 33,3 & - & 6,2 & 1,8 & - & 100,0 & - & 50,0 & 50,0 & 50,0 & 44,4 \\
\hline & Outros & 20,0 & 20,0 & 60,0 & 16,7 & 36,4 & 31,2 & 28,6 & 85,7 & - & 60,0 & 66,7 & 77,8 & 46,1 & 58,2 & 100,0 & 100,0 & 100,0 & 100,0 & 100,0 & 100,0 & 100,0 \\
\hline \multirow{4}{*}{ Prevenção } & Lavar as mãos & 13,3 & 50,0 & - & - & 9,1 & 6,2 & 14,3 & 42,8 & - & - & 16,7 & 22,2 & 15,4 & 20,0 & - & - & 100,0 & 50,0 & 50,0 & - & 33,3 \\
\hline & $\begin{array}{l}\text { Evitar contato } \\
\text { com solo }\end{array}$ & 13,3 & 60,0 & - & - & 18,2 & 12,5 & 19,0 & 21,4 & - & - & 16,7 & 22,2 & 15,4 & 14,5 & - & - & - & 50,0 & - & 50,0 & 22,2 \\
\hline & Higiene & 13,3 & - & 80,0 & - & - & - & 9,5 & 28,6 & 12,5 & 60,0 & - & - & 30,8 & 21,8 & 100,0 & 100,0 & - & - & 50,0 & 50,0 & 44,4 \\
\hline & Outros & 26,7 & 20,0 & 100,0 & 16,7 & 54,5 & 37,5 & 38,1 & 42,8 & 50,0 & 100,0 & 33,3 & 55,5 & 23,1 & 45,4 & - & 100,0 & 100,0 & 100,0 & 50,0 & 50,0 & 66,7 \\
\hline \multicolumn{2}{|c|}{ № Total de participantes } & 15 & 10 & 5 & 6 & 11 & 16 & 63 & 14 & 8 & 5 & 6 & 9 & 13 & 55 & 1 & 1 & 1 & 2 & 2 & 2 & 9 \\
\hline
\end{tabular}

Fonte: Elaborado pelos autores 


\section{Representações dos responsáveis}

A maioria dos responsáveis (30,9\%) não soube responder o conceito de vermes e 29,1\% responderam incorretamente. Esses dados corroboram com a pesquisa de Rodrigues et al. (2018) em Grajaú - MA, onde 63,6\% dos pais não souberam responder o que são parasitos. Nesse sentido, Santos, Costa e Castro (2017) relatam que o desconhecimento dos responsáveis, contribui para o aumento de riscos de infecções nas crianças, uma vez que as primeiras instruções são recebidas no ambiente familiar. A outra parcela dos entrevistados respondeu parcialmente correto $(23,6 \%)$ e correto $(16,4 \%)$, mencionando ameba $(16,4 \%)$, giárdia $(14,5 \%)$, lombriga e parasita (12,7\% cada) e bicho (10,9\%). Este conhecimento pode estar associado as informações recebidas através de diagnósticos médicos e/ou devido as crianças expelirem o parasito, pois de acordo com Pereira et al. (2018), Entamoeba histolytica Schaudinn, 1903, Giardia lamblia Ancey, 1906 e A. lumbricoides estão entre os parasitos mais prevalentes entre escolares.

Quanto às formas de transmissão, a maioria dos responsáveis não soube responder $(60,0 \%)$ e outros responderam incorretamente (5,4\%), assim, podemos inferir que a falta de conhecimento dos responsáveis, pode constitui um fator de risco para ocorrências de infecções parasitárias. Os demais participantes responderam parcialmente correto $(7,3 \%)$ e correto $(27,3 \%)$, citando alimentos contaminados $(20,0 \%)$, contato com solo $(9,0 \%)$ e outras formas de infecção (27,3\%), não tecendo quaisquer relações das vias de transmissão com as doenças que podem ser causadas. Diferentemente destes resultados, em uma pesquisa na área rural da Abaye Deneba - Etiópia (África), Nyantekyi et al. (2014), observaram que os moradores conheciam as vias de transmissão e conseguiam correlaciona-las com as doenças transmitidas, a exemplo de alimentos contaminados como causa de ascaridíase (10,2\%), carne crua para teníase (14,5\%) e água contaminada para esquistossomose (18,3\%), amebíase $(24,4 \%)$ e giardíase (59,3\%).

Com relação ao habitat e aos sintomas, 69,1\% responderam corretamente sobre ambos. Quanto as localizações, as mais citadas foram intestino grosso e delgado (65,4\% cada), cérebro $(5,4 \%)$ e ânus (5,4\%). Além destas, 30,9\% mencionaram outros locais, como fígado, coração, rins e pulmões. Sobre os sintomas, foi relatado principalmente dor de barriga (36,4\%), seguido por falta de apetite $(25,4 \%)$, vômito $(23,6 \%)$, manchas $(16,4 \%)$ e dor na cabeça (3,6\%). De modo similar, Busato et al. (2015) ao investigar as percepções de uma comunidade 
de Santa Catarina verificaram que o habitat e sintoma mais citado foi o intestino (68\%) e dor de barriga (30\%), respectivamente.

Sobre as medidas de prevenção, 9,1\% responderam parcialmente correto, 3,6\% incorretamente e 36,4\% não souberam responder. Enquanto, 50,9\% responderam corretamente, mencionando ter higiene $(21,8 \%)$, lavar as mãos $(20,0 \%)$, evitar contato com o solo $(14,5 \%)$ e outras formas de prevenir $(45,4 \%)$. Com base nesses dados podemos verificar que as práticas preventivas expressadas pelos responsáveis são pouco específicas com relação aos hábitos higiênicos, pois não relatam como lavar as mãos ou o que utilizam para lava-las.

Na pesquisa de Forson, Arthur e Ayeh-Kumi (2018), ao investigarem o papel da educação dos pais na prevalência de infecções parasitárias em escolares em Acra - Gana (África), constataram que $93,7 \%$ dos pais das crianças infectadas não tinham qualquer conhecimento sobre as parasitoses intestinais. Nesse contexto, Gniadek et al. (2015) inferem que a ausência de conhecimento dos pais acerca das medidas preventivas, torna seus filhos mais susceptíveis a adquirir infecções, e por terem influência nos hábitos dos filhos, devem contribuir com ensinamentos corretos para profilaxia frente as doenças parasitárias.

\section{Representações dos docentes}

Quanto ao conceito de vermes, 55,6\% dos docentes responderam corretamente e 22,2\% parcialmente correto, citando lombriga e doença (55,6\% cada), taenia e parasita (44,4\% cada), ameba (33,3\%) e giárdia (22,2\%). Sá-Silva et al. (2010) realizaram um estudo com quatro professoras de uma escola municipal de São Luís - MA, e o termo lombriga foi mencionado por todas elas como agente causador das verminoses. Segundo os autores a grande utilização deste vocábulo pode estar associada as campanhas educacionais, a ênfase nos livros didáticos e a alta prevalência de A. lumbricoides a nível mundial.

Sobre a transmissão, 66,7\% responderam corretamente, sendo citado alimentos contaminados (55,5\%), contato com o solo $(33,3 \%)$ e outros $(66,7 \%)$ mecanismos de contaminação, como falta de higiene, carne malcozida, água contaminada e ausência de saneamento básico. Similarmente, no estudo de Monroe et al. (2013), as professoras de São 
Luís - MA elencaram como principais formas de transmissão a falta de higiene, contato com solo, falta de saneamento básico, água e alimentos contaminados. Os autores ressaltam que devido as docentes terem participação na construção de condutas, é de suma relevância que conheçam as vias de contaminação para difundi-las entre os discentes, almejando a redução dos riscos de infecção parasitária.

A respeito do habitat dos parasitos, 55,6\% responderam correto e 33,3\% parcialmente correto, as respostas mais mencionadas foram, intestino grosso e delgado (77,7\%, cada), cérebro $(33,3 \%)$ e ânus $(11,1 \%)$. Todos os docentes mostraram conhecer os sintomas das parasitoses intestinais, citando falta de apetite $(66,7 \%)$, manchas $(44,4 \%)$ e vômito $(22,2 \%)$. Referente a prevenção, 88,9\% dos docentes responderam corretamente, mencionando ter higiene (44,4\%), lavar as mãos (33,3\%), evitar contato com o solo $(22,2 \%)$ e outras medidas $(66,7 \%)$ como tratar a água, lavar os alimentos e tomar banho. Resultados semelhantes foram reportados por docentes nas pesquisas de Metuh e Ikpeze (2009) e Njomo et al. (2016), os quais informaram lavar as mãos e alimentos, usar calçado, e consumir água tratada. Diferente deste estudo, nos trabalhos anteriores, foram ainda mencionados a utilização do sanitário, a importância do saneamento ambiental e a educação em saúde.

\section{docentes}

Correlação entre as representações da tríplice: discentes, responsáveis e

Quanto ao conceito e nomeação dos parasitos, a maior parte dos docentes apresentaram conhecimento adequado, ao contrário da maioria dos discentes e responsáveis. Assim, existe a possibilidade de os discentes terem recebido informações em sala de aula e não terem assimilado o conteúdo, como também, o docente não ter repassado as informações adquiridas. Grimes, Ronchi e Hirano (2013) relatam que temas transversais como a educação para a saúde são inclusos nos currículos, no entanto, conteúdos referentes a parasitologia apresentam-se segmentados na grade curricular de ciências, e na maioria das vezes são pouco aprimorados ou deixam até mesmo de serem trabalhados. Nesse sentido, Monroe et al. (2013) informam que há a necessidade de mudanças efetivas no processo de formação dos docentes, afim de potencializar os ensinamentos referentes a educação em saúde. 
Com relação a transmissão, a tríplice apresentou desconhecimento e respostas pouco específicas, alertando assim, o alto risco de infecção o qual os participantes, especialmente os discentes podem estar expostos, considerando que os responsáveis e docentes constituem os principais canais de informações. É válido destacar que poucos entrevistados relataram as vias de transmissão que podem ocasionar infecções por parasitos específicos, como: Schistosoma mansoni (Sambon, 1970) ao ter contato com água contaminada; Taenia sp. Linnaeus, 1758 pelo consumo de carne de boi ou porco malcozida e A. lumbricoides, E. histolytica, bem como, G. lamblia por ingestão de água e/ou alimentos contaminados (ANDRADE et al., 2010).

Malafaia et al. (2013) ressaltam que é importante entender a dinâmica de transmissão dos parasitos, pois auxilia no melhoramento e estabelecimento de atitudes profiláticas frente às parasitoses. Consoante a isso, no trabalho de Njomo et al. (2016), foi verificado que os professores reconhecem que devem atuar informando as crianças sobre os meios de transmissão, bem como, estar envolvidos afim de sensibilizar toda a sociedade. O consumo de doces, foi a resposta equivocada mais citada como forma de adquirir parasitos, por discentes (17,5\%), responsáveis $(5,4 \%)$ e docentes $(11,1 \%)$. Bath et al. (2010) inferem que os responsáveis pelas crianças tentam convence-las do mal que os doces fazem à saúde, propagando a informação errônea de que os parasitos são transmitidos através de doces. De acordo com Moura et al. (2018) essas informações estão conexas a divulgação deste mito no espaço informal, devido ao quadro diarreico que pode ocorrer em algumas crianças quando ingerem uma grande quantidade de doces, assim como ocorre nas infecções por vermes.

Acerca do habitat dos parasitos e dos sintomas causados por eles, pode-se observar que as respostas dos discentes, responsáveis e docentes que responderam adequadamente, foram semelhantes, sendo as respostas mais citadas o intestino e dor de barriga. Esses dados assemelham-se ao estudo realizado por Siqueira et al. (2016) entre o público adulto e infantil. Sobre a prevenção, houve um consenso de respostas corretas entre toda a tríplice, onde as ações mais citadas foram lavar as mãos e os alimentos, evitar contato com o solo e ter higiene. Porém nenhum dos entrevistados especificaram as respostas. Outras atitudes preventivas essenciais foram pouco reportadas, como ingerir água potável, cortar as unhas, lavar as mãos 
antes das refeições e após utilizar o banheiro e/ou como lavá-las (ZAIDEN et al., 2008; SANTOS; GURGEL-GONÇALVES; MACHADO, 2014).

Referente ao cuidado com os alimentos, Souza et al. (2016) relatam que é importante utilizar soluções higienizadoras para lava-los, a exemplo: hipoclorito de sódio, vinagre ou simplesmente água fervida. Uma vez que, hortaliças e carnes são alimentos geralmente adquiridos em feiras livres e ficam expostas a insetos e poeira, que podem transportar ovos de parasitos (FERNANDES et al., 2015). Desse modo, torna-se perceptível a necessidade do trabalho conjunto com a família, tendo em vista que são fortes influenciadores na formação de opinião entre os discentes (SÀ-SILVA et al., 2010). E assim, tornar a educação um processo de constante acontecimento, formal ou informal, contanto que haja uma relação entre o que é ensinado na escola e em casa, principalmente para a prevenção de doenças como as parasitoses intestinais (MONROE et al., 2013).

\section{CONSIDERAÇÕES FINAIS}

Os dados apresentados destacam a importância de investigar simultaneamente as representações de discentes, docentes e familiares sobre parasitoses intestinais, para melhor compreender a influência que as concepções sociais, exercem nos casos de adoecimento da população infantil. Assim, foi possível identificar que os docentes e responsáveis apresentaram lacunas e equívocos, especialmente sobre as vias de transmissão e medidas profiláticas, que podem ter refletido no desconhecimento dos escolares sobre estes aspectos. Desse modo, se faz necessário o fomento a formação continuada de professores no âmbito da educação em saúde, bem como, da necessidade de atividades didático-pedagógicas que promovam ações entre escola e família, visando estimular mudanças comportamentais, reduzir os riscos de infecções e melhorar a qualidade de vida individual e coletiva.

\section{REFERÊNCIAS}

AHMED, K. S.; SIRAJ, N. M.; FITSUMBERHAN, H.; ISAAC, S.; YOHANNES, S.; EMAN, D.; BERHANE, Y.; ARAYA, M. Knowledge, Attitude and Practice (KAP) Assessment of Intestinal Parasitic Infection among School Children in Asmara, Eritrea, Saúde, $\quad$ v. $\quad 9, \quad$ n.1, p.57-68, 2017. Disponível em <https://www.scirp.org/journal/paperinformation.aspx?paperid=73419>. Acesso em 17 de setembro de 2019. 
ALBUQUERQUE, U. P. (Org.). Introdução à Etnobiologia. Recife, PE: NUPEEA, 2014. 189 p.

ALMEIDA, N. R. B.; CARVALHO, A. S.; MORENO, C. A. Análise Parasitológica de contaminantes de origem fecal encontrados em uma Creche do interior da Bahia. Id on Line Revista Multidisciplinar e de Psicologia, v. 11, n. 38, p. 642-651, 2017. Disponível em: <https://idonline.emnuvens.com.br/id/article/view/930>. Acesso em 22 de setembro de 2019 .

ALVES, Paulo César. Itinerário terapêutico e os nexus de significados da doença. Revista de Ciências Sociais, $n$. 42, p. 29-43, 2015.2 Disponível em: <http://www.siga.fiocruz.br/arquivos/ss/documentos/editais/4_artigo2\%20alves\%20paulo\%20cesar.pdf>. Acesso em 25 de setembro de 2019.

ANDRADE, E. C.; LEITE, I. C. G.; RODRIGUES, V. O.; CESCA, M. G. Parasitoses Intestinais: Uma revisão sobre seus Aspectos Sociais, Epidemiológicos, Clínicos e Terapêuticos. Revista de APS, v. 13, n. 2, p. 231-240, 2010. Disponível em: <https://periodicos.ufjf.br/index.php/aps/article/view/14508>. Acesso em 01 de outubro de 2019.

BARBOSA, R. N.; MOTA, B. A. E.; CAVALCANTI, M. D. B.; BOTÊLHO, M. D. C. N.; SANTOS, E. M. Enteroparasitas e profilaxia em alunos da zona rural de Serra Talhada-Pernambuco. Biológicas \& Saúde, v. 3, n. 9, p. 37-45, 2013. Disponível em: <https://pdfs.semanticscholar.org/86fe/daeb953a6d865368088ce9ae9a4b1464fb8c.pdf>. Acesso em 26 de outubro de 2019.

BATH, J. L.; ENEH, P.N.; BAKKEN, A. J.; KNOX, M. E.; SCHIEDT, M. D.; CAMPBELL, J. M. The impact of Perception and Knowledge on the treatment and Prevention of intestinal Worms in the Manikganj district of Bangladesh. Journal of Biology and Medicine, v.83, n.4, p.171-184, 2010. Disponível em: <https://www.ncbi.nlm.nih.gov/pmc/articles/PMC3002153/>. Acesso em 28 de outubro de 2019.

BERNARD, H. R. Research methods in anthropology: qualitative and quantitative approaches. 4 ed. New York: AltaMira Press, 2006. 803 p.

BUSATO, M. A.; DONDONI, D. Z.; RINALDI, A. L. S.; FERRAZ, L. Parasitoses intestinais: O que a comunidade sabe sobre este tema?. Revista Brasileira de Medicina de Família e Comunidade, v. 10, n. 34, p. 1-6, 2015. Disponível em: <https://www.rbmfc.org.br/rbmfc/article/view/922>. Acesso em 23 de setembro de 2019.

BRAGAGNOLLO, G. R.; TOLEDO, P. C. G.; MORERO, J. A. P.; SCARPINI, N. A. M.; FERREIRA, B. R. Avaliação de um programa educativo sobre parasitoses intestinais implementado em uma comunidade escolar pelo olhar do professor. Revista Uningá, v. 51, n. 1, 2017. Disponível em: <http://revista.uninga.br/index.php/uninga/article/view/1343>. Acesso em 21 de setembro de 2019.

BRAGAGNOLLO, G. R.; GODOY, P. C. G. D. T.; SANTOS, T. S. D.; RIBEIRO, V. D. S.; MORERO, J. A. P.; FERREIRA, B. R. Intervenção educacional sobre enteroparasitoses: um estudo quase experimental. Revista Cuidarte, v. 9, n. 1, p. 2030-2044, 2018. Disponível em: <http://www.scielo.org.co/scielo.php?pid=S221609732018000102030\&script=sci_abstract\&tlng=en>. Acesso em 20 de setembro de 2019.

COSTA, E. L.; SOUZA, J. R. S. FAMÍLIA E ESCOLA: AS CONTRIBUIÇÕES DA PARTICIPAÇÃO DOS RESPONSÁVEIS NA EDUCAÇÃO INFANTIL. Khóra: Revista Transdisciplinar, v. $6, \quad$ n. $\quad 7, \quad 2019 . \quad$ Disponível em: <http://www.site.feuc.br/khora/index.php/vol/article/viewFile/166/113>. Acesso em 29 de outubro de 2019.

FARIA, K. F., MOTA, K. C. P., SILVA, C. O., DE OLIVEIRA, M. M., ARAÚJO, I. A. C., MENDES, G. G.; PEREIRA, A. D.; CURY, M. C. Ensino em parasitologia: Ação extensionista com crianças em idade escolar. Revista Conexão UEPG, v. 15, n. 3, p. 294-300, 2019. Disponível em: <https://dialnet.unirioja.es/servlet/articulo?codigo=7052754>. Acesso em 02 de novembro de 2019.

FERNANDES, N. S.; GUIMARAES, H. R.; AMORIM, A. C. S.; REIS, M. B.; TRINDADE, R. A.; MELO, A. C. F. L. Avaliação parasitológica de hortaliças: da horta ao consumidor final. Revista Saúde e Pesquisa, v. 8, n. 2, p. 255-265, 2015. 
Disponível em: <https://periodicos.unicesumar.edu.br/index.php/saudpesq/article/view/4174>. Acesso em 21 de dezembro de 2019.

FONSECA, E. O. L.; TEIXEIRA, M. G.; BARRETO, M. L.; CARMO, E. H.; COSTA, M. D. C. N. Prevalência e fatores associados às geo-helmintíases em crianças residentes em municípios com baixo IDH no Norte e Nordeste brasileiros. Cadernos de Saúde Pública, v. 26, n. 1, p. 143-152, 2010. Disponível em: <https://www.scielosp.org/article/csp/2010.v26n1/143-152/>. Acesso em 23 de dezembro de 2019.

FORSON, A. O.; ARTHUR, I.; AYEH-KUMI, P. F. The role of family size, employment and education of parents in the prevalence of intestinal parasitic infections in school children in Accra. PloS one, v. 13, n. 2, p. 1-10, 2018. Disponível

em: <https://journals.plos.org/plosone/article/file?type=printable\&id=10.1371/journal.pone.0192303>. Acesso em 19 de dezembro de 2019.

GAZZINELLI, M. F.; GAZZINELLI, A.; REIS, D. C. D.; PENNA, C. M. D. M. Educação em saúde: conhecimentos, representações sociais e experiências da doença. Cadernos de Saúde Pública, v. 21, p. 200-206, 2005. Disponível em: <https://www.scielosp.org/article/csp/2005.v21n1/200-206/>. Acesso em 20 de setembro de 2019.

GNIADEK, A.; CEPUCH, G.; OCHENDER, K.; SALAMON, D. The level of knowledge about parasitic diseases and the threats resulting from their presence in the environment evaluated in a group of parents of preschool children. Annals of parasitology, $\quad$ v. 61, n. 2, p. 109-114, 2015. Disponível em: <http://yadda.icm.edu.pl/yadda/element/bwmeta1.element.agro-d0acc3a6-3ef2-412f-af5e-e4a419ba414e>. Acesso em 18 de dezembro de 2019.

GRIMES, C.; RONCHI, D. L.; HIRANO, Z. M. B. Prática pedagógica diferenciada nos processos de ensinar e de aprender em parasitologia. Ensino, Saúde e Ambiente, v. 6, n. 1, p. 89-100, 2013. Disponível em: $<$ https://periodicos.uff.br/ensinosaudeambiente/article/view/21007>. Acesso em 05 de janeiro de 2020.

IBGE. (Instituto Brasileiro de Geografia e Estatística). Cidades: Bahia: Paulo Afonso: Infográficos: evolução populacional e pirâmide etária. 2010. Disponível em: <https://cidades.ibge.gov.br/brasil/ba/pauloafonso/panorama>. Acesso em 10 de agosto de 2019.

LOPES, R. M.; MELO, T. L. Percepção dos alunos, em anos iniciais do Ensino Fundamental, relacionada à higienização das mãos. Revista Eletrônica Interdisciplinar, v. 1, n. 11, p. 117-121, 2014. Disponível em: $<$ https://silo.tips/download/interdisciplinar-revista-eletronica-da-univar-issn-X-ano-de-publicaao-2014-n11v>. Acesso em 27 de setembro de 2019.

MALAFAiA, G.; GONÇALVES, R. C.; FALEIRO, J. H.; DA SILVA CASTRO, A. L.; DE LIMA RODRIGUES, A. S. Conhecimentos de Discentes do Ensino Fundamental e Médio de uma Escola Pública de Urutaí (Goiás) Sobre Doenças Intestinais. Saúde e Pesquisa, v. 6, n. 2, p. 237-247, 2013. Disponível em: $<$ https://periodicos.unicesumar.edu.br/index.php/saudpesq/article/view/2781>. Acesso em 29 de setembro de 2019.

MELLO, D. A.; PRIPAS, S.; FUCCI, M.; SANTORO, M. C.; PEDRAZZANI, E. S. Helmintoses intestinais: IConhecimentos, atitudes e percepção da população. Revista de Saúde Pública, v. 22, n. 2, p. 140-149, 1988. Disponível em: <https://www.scielosp.org/article/rsp/1988.v22n2/140-149/pt/>. Acesso em 18 de setembro de 2019.

MINISTÉRIO DA EDUCAÇÃO. Diretrizes Curriculares Nacionais Gerais da Educação Básica. Brasília: Ministério da Educação. Secretaria de Educação Básica. Diretoria de Currículos e Educação Integral, 2013, 565p. Disponível em: <http://portal.mec.gov.br/docman/julho-2013-pdf/13677-diretrizes-educacao-basica-2013pdf/file>. Acesso em 29 de agosto de 2019.

METUH, O. A.; IKPEZE, O. O. Knowledge, attitude and practice (Kap) of school teachers on malaria, helminthiasis and associated risk factors in primary schools in Onitsha, Anambra state, South-Eastern Nigeria. Animal Research International, v. 6, n. 2, p. 987-993, 2009. Disponível em: $<$ https://www.ajol.info/index.php/ari/article/view/48130>. Acesso em 15 de janeiro de 2020. 
MONROE, N. B.; LEITE, P. R. R.; SANTOS, D. N.; SÁ-SILVA, J. R. O tema transversal saúde e o ensino de ciências: representações sociais de professores sobre as parasitoses intestinais. Investigações em Ensino de Ciências, v. 18, n. 1, p. 7-22, 2013. Disponível em: <https://www.if.ufrgs.br/cref/ojs/index.php/ienci/article/view/146>. Acesso em 24 de setembro de 2019.

MONTEIRO, A. M. C.; SILVA, E. F.; ALMEIDA, K. S.; SOUSA, J. J. N.; MATHIAS, L.A.; BAPTISTA, F.; FREITAS, F. L. C. Parasitoses intestinais em crianças de creches públicas localizadas em bairros periféricos do munícipio de Coari, Amazonas, Brasil. Revista de Patologia Tropical, v. 38, n. 4, p. 284-290, 2009. Disponível em: <http://files.cercomp.ufg.br/weby/up/63/o/2009_38_4_284_290.pdf>. Acesso em 28 de setembro de 2019.

MOSCOVICI, S. A representação social da psicanálise. Traduzido por Álvaro Cabral. Rio de Janeiro: Zahar, 1978.

MOURA, M. Q.; JESKE, S. T.; ALMEIDA CAPELLA, G.; PINTO, N. B.; BIANCHI, T. F.; BERNE, M. E. A.; VILLELA, M. M. Percepções de escolares da cidade de Pelotas/RS sobre parasitoses intestinais. SaBios-Revista de Saúde e Biologia, v. 13, n. $\quad 1, \quad$ p. $56-62, \quad 2018 . \quad$ Disponível em: $<$ http://revista2.grupointegrado.br/revista/index.php/sabios2/article/view/2555>. Acesso em 19 de outubro de 2019.

NEVES, D. P. Parasitologia Dinâmica. 3.ed., São Paulo: Editora Atheneu, 2009, p. 592.

NEVES, D. P; FILIPPIS, T.; DIAS-LIMA, A.; ODA, W. Y. Parasitologia Básica. 4ª .ed. Editora Atheneu, 2018, p. 256.

NJOMO, D. W.; MASAKU, J.; ODHIAMBO, G.; MUSUVA, R.; MWENDE, F.; MATEY, E.; KIHARA, J. H. The role of preschool teachers in the control of soil-transmitted helminthes in coastal region, Kenya. Tropical diseases, travel medicine and vaccines, v. 2, n. 1, p. 24, 2016. Disponível em: <https://link.springer.com/article/10.1186/s40794-016-0040-y>. Acesso em 21 de janeiro de 2020.

NUNES, A. L. B. P.; CUNHA, A. M. O.; MARÇAL JÚNIOR, O. Coletores de lixo e enteroparasitoses: o papel das representações sociais em suas atitudes preventivas. Ciência \& Educação. p. 25-38, 2006. Disponível em: $<$ https://www.scielo.br/scielo.php?script=sci_arttext\&pid=\$1516-73132006000100004>. Acesso em 22 de setembro de 2019.

NYANTEKYI, L.; LEGESSE, M.; MEDHIN, G.; ANIMUT, A.; TADESSE, K.; MACIAS, C.; ERKO, B. Community awareness of intestinal parasites and the prevalence of infection among community members of rural Abaye Deneba area, Ethiopia. Asian Pacific journal of tropical biomedicine, v. 4, n. 1, p. 152-157, 2014. Disponivel em: $<$ https://www.sciencedirect.com/science/article/pii/S2221169115302598>. Acesso em 20 de janeiro de 2020.

OLIVEIRA, A. S. D. S. S.; SILVA, B. E. S.; CUNHA, E. A.; MACEDO, J. L.; RODRIGUES, A. C. E.; ASSUNÇÃO, M. D. J. S. M.; AZEVÊDO, C. A. S. Enteroparasitoses in schools of the municipal public network. Research, Society and Development, v. $8, \quad$ n. 4 , p. 384-955, 2019. Disponível em: $<$ https://rsd.unifei.edu.br/index.php/rsd/article/view/955>. Acesso em 12 de janeiro de 2020.

PEREIRA, G.; RIBEIRO, C.; COSTA, I.; SILVA, J.; CALADO, L.; NUNES, B.; MOTA, M. Prevalência de infecções parasitárias intestinais oriundas de crianças residentes em áreas periféricas, município de Juazeiro do NorteCeará. Revista Interfaces: Saúde, Humanas e Tecnologia, v. 5, n. 14, p. 21-27, 2018. Disponível em: $<$ http://interfaces.leaosampaio.edu.br/index.php/revista-interfaces/article/view/587>. Acesso em $10 \mathrm{de}$ janeiro de 2020.

PREFEITURA MUNICIPAL DE PAULO AFONSO. Cidade: Aspectos, população, relevo, clima e vegetação. 2014. Disponível em: <http://www.pauloafonso.ba.gov.br/novo/?p=turismo\&i=3>. Acesso em 10 de março de 2019.

RIVERO, M. R.; SALAS, M. M.; VALENTE, R.; NORES, M. J.; DE ANGELO, C.; ARRABAL, J.; SALOMÓN, O. D. Prevention of intestinal parasites in a tri-border area of Latin America: Children perceptions and an integral health education strategy.Zoonoses and public health, v. 64, n. 8, p. 673-683, 2017. Disponível em: 
<https://onlinelibrary.wiley.com/doi/full/10.1111/zph.12365?casa_token=tnZMD5gD0AAAAAA\%3A3zZCyPhA5wpUhPrespjYO95UxbehWiW_PW3MuIBnjTgEFzbLCM5AuSKhjuA6YBZ7lPos_FnlP_7Eg>. Acesso em 17 de janeiro de 2020.

RODRIGUES, S. R.; GOMES, S. C. S.; LIMA, R. J. C. P.; NASCIMENTO, J. X. P. T. Projeto Parasitoses Intestinais em crianças: prevalência e fatores associados. Revista Ciência em Extensão, v. 14, n. 3, p. 64-78, 2018. Disponível em:< https://ojs.unesp.br/index.php/revista_proex/article/view/1587>. Acesso em 20 de janeiro de 2020.

SANTOS, A. A.; GURGEL-GONÇALVES, R.; MACHADO, E. R. Factors associated with the occurrence of intestinal parasites in children living in the Federal District of Brazil. Revista de Patologia Tropical/Journal of Tropical Pathology, v. 43, n. $1, \quad$ p. 89-97, 2014. Disponível em: <https://pdfs.semanticscholar.org/c82e/6bf2cd23b6650f212b3a19b97f6621120022.pdf>. Acesso em 16 de janeiro de 2020.

SÁ-SILVA, J. R.; PORTO, M. J. F.; SOUSA, C. E. B.; ALMEIDA, F. V. P. Escola, educação em saúde e representações sociais: Problematizando as parasitoses intestinais. Pesquisa em Foco, v. 18, n. 1, p. 82-95, 2010. Disponível em: <http://45.71.6.41/index.php/PESQUISA_EM_FOCO/article/view/325>. Acesso em 23 de setembro de 2019.

SANTOS, D. N.; COSTA, P. R. R. S.; CASTRO, N. J. C. Saberes sobre Parasitoses em uma Comunidade Riberinha. Revista Interdisciplinar de Estudos em Saúde, v.6, n. 2, p. 44-56, 2017. Disponível em: $<$ http://periodicos.uniarp.edu.br/index.php/ries/article/view/1071>. Acesso em 22 de janeiro de 2020.

SILVA, E. P.; CARVALHO, W. R. C.; FIRMO, W. C. A. Estudo comparativo da ocorrência de parasitoses intestinais em crianças de duas comunidades da zona rural de Paulo Ramos -MA, Brasil. Revista UNINGÁ. v. 27, n. 2, p. 15-21, 2016. Disponível em: 〈http://34.233.57.254/index.php/uningareviews/article/view/1824>. Acesso em 29 de janeiro de 2020.

SIQUEIRA, M. P.; AZEVEDO, E. P.; ALMEIDA, E.M.; MATOS, J. S.; RODRIGUES, A. R.; SCARABELLI, S. C.; PILOTTO, T. P.; FREITAS, J. T.; BARBOSA, A. S.; MATTOS, D. P. B. G.; BASTOS, O. M. P.; UCHÔA, C. M. A. Conhecimentos de escolares e funcionários da Rede Pública de Ensino sobre as parasitoses intestinais. Revista Instituto Adolfo Lutz, v. 75, n. 0, p. 01-12, 2016. Disponível em: <https://pesquisa.bvsalud.org/portal/resource/pt/biblio835643>. Acesso em 27 de setembro de 2019.

SOUZA, A. C.; ALVES, F. V.; GUIMARÃES, H. R.; AMORIM, A. C. S.; DE ARAÚJO CRUZ, M.; DA SILVA SANTOS, B.; MELO, A. C. F. L. Perfil epidemiológico das parasitoses intestinais e avaliação dos fatores de risco em indivíduos residentes em um assentamento rural do nordeste brasileiro. Revista Conexão UEPG, v. 12, n. 1, p. 26-37, 2016. Disponível em: $<$ https://dialnet.unirioja.es/servlet/articulo?codigo=6860660>. Acesso em 28 de janeiro de 2020.

VIVAS, A.; GELAYE, B.; ABOSET, N.; KUMIE, A.; BERHANE, Y.; WILLIAMS, M. A. Knowledge, attitudes, and practices (KAP) of hygiene among school children in Angolela, Ethiopia. Journal of preventive medicine and hygiene, v. 51, n. 2, p. 73, 2010. Disponível em: <https://pubmed.ncbi.nlm.nih.gov/21155409/>. Acesso em 29 de janeiro de 2020 .

ZAIDEN, M. F.; SANTOS, B. O.; CANO, M. A. T.; JÚNIOR, I. A. N. Epidemiologia das parasitoses intestinais em crianças de creches de Rio Verde - GO. Medicina, v. 41, n. 2, p. 182-187, 2008. Disponível em: $<$ http://revista.fmrp.usp.br/2008/VOL41N2/ao_parasitoses_intestinais_criancas_creches_rio_verde.pdf>. Acesso em 27 de janeiro de 2020.

\section{(cc) $\mathrm{EY}$}

Este trabalho está licenciado com uma Licença Creative Commons - Atribuição 4.0 Internacional. 